\title{
Norois
}

Environnement, aménagement, société

$227 \mid 2013$

Sentir et ressentir la ville

\section{Introduction. Une ville, cinq sens, trois traitements : sensoriel, cognitif et affectif}

Introduction. A City, Five Senses, Three Treatments: Sensorial, Cognitive and Emotional

\section{Denis Martouzet}

\section{OpenEdition}

Journals

Édition électronique

URL : http://journals.openedition.org/norois/4635

DOI : $10.4000 /$ norois. 4635

ISBN : 978-2-7535-2916-8

ISSN : $1760-8546$

Éditeur

Presses universitaires de Rennes

\section{Édition imprimée}

Date de publication : 30 juin 2013

Pagination : $7-10$

ISBN : 978-2-7535-2882-6

ISSN : 0029-182X

\section{Référence électronique}

Denis Martouzet, «Introduction. Une ville, cinq sens, trois traitements : sensoriel, cognitif et affectif», Norois [En ligne], 227 | 2013, mis en ligne le 30 juin 2013, consulté le 22 septembre 2020. URL : http:// journals.openedition.org/norois/4635; DOI : https://doi.org/10.4000/norois.4635

(c) Tous droits réservés 


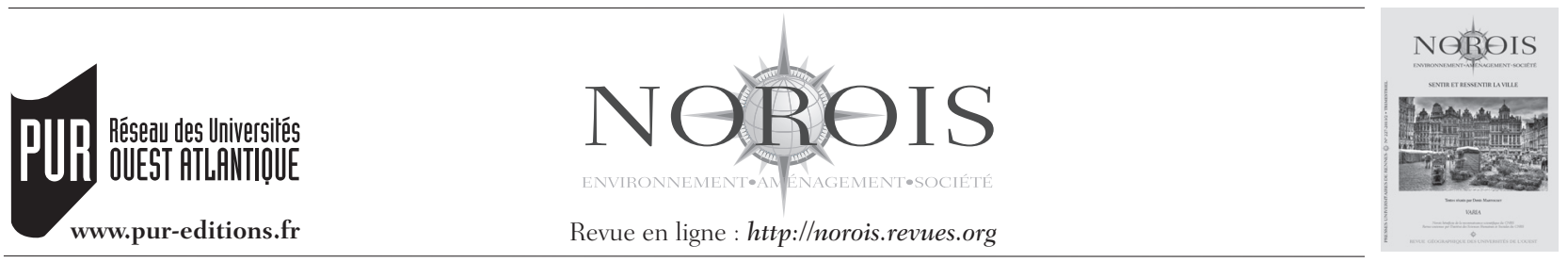

\title{
Introduction \\ Une ville, cinq sens, trois traitements : sensoriel, cognitif et affectif
}

\author{
Denis Martouzet
}

IPAPE - UMR 6173 CITERES, Université de Tours, 33-35, allée Ferdinand-de-Lesseps - 37200 Tours (denis. martouzet@univ-tours.fr)

Sentir, ressentir la ville : cela pourrait apparaître, au premier abord, comme un simple jeu de mots, autour de leur sonorité et de leur proximité de sens. Sentir renvoie d'abord, spécifiquement à l'odorat mais aussi, plus largement, à la sensorialité, la capacité qu'ont nos sens de nous approcher du réel qui nous entoure, et à la sensibilité, c'est-à-dire la plus ou moins grande force avec laquelle nos sens nous procurent des sensations. Par ailleurs, la sensiblerie semble être l'exagération de cette sensibilité, qu'on dira alors à fleur de peau ou exacerbée. Mais, de ce fait, il apparaît évident que le traitement de l'information que nous apporte les sens ne relèvent pas que de l'objet senti (sa couleur, sa forme, son odeur, sa sonorité, son goût, sa rugosité...), ni que de la dimension physique et chimique de nos organes sensoriels. Il s'agit aussi d'un traitement neurologique et psychologique, sans qu'il y ait nécessairement sensiblerie qui convoque l'entièreté de la personne, y compris ses sentiments. Ce qui est senti est toujours déjà en même temps ressenti.

La confusion possible liée à la proximité de sens est amplifiée par le flou sémantique de chacun de ces termes, l'imprécision du vocabulaire. On a noté, à travers l'exemple du verbe sentir, la plurivocité de certains de ces termes : sentir réfère au seul odorat comme à l'ensemble des sens sans que pour autant on puisse l'associer à chacun d'eux. Le caractère flou est le résultat de la complexité des phénomènes et mécanismes physiques, chimiques, corporels, neurologiques et psychologiques en œuvre subsumés par ces mots. De façon synthétique, toutes les contributions qui suivent le montrent ou sont soustendus par l'idée que sentir c'est déjà ressentir.

Préalablement, il paraît fondé d'insister sur les liens qui existent entre les sens, pris d'abord dans leur seule dimension corporelle. Il y a soit, dans la simultanéité, renforcement de l'impression produite comme c'est le cas pour l'odorat et le goût (les personnes ayant moins d'odorat goûtent moins les aliments), soit préparation d'un sens à sentir par ce qu'un autre a perçu : deviner l'aliment goûté sans l'avoir vu ou, plus globalement, sans avoir à l'avance d'indication sur ce que c'est se révèle plus difficile qu'on ne le croit, conduire une voiture suppose l'écoute qui prévient, dans certains cas, du danger éventuel, confirmé ou non par la vue. Il y a alors consécutivité et non plus simultanéité, via le système neurophysiologique. 
Certains estiment que les sens peuvent être hiérarchisés, ce qui reste discutable. En revanche, il est clair que leurs modes de fonctionnement respectifs, purement sensoriels comme en lien avec ce que les sens convoquent ou permettent de mobiliser, sont différents. Marcel Proust, à sa manière, dans À la recherche du temps perdu, magnifie le goût et l'odorat : « La vue de la petite madeleine ne m'avait rien rappelé avant que je n'y eusse goûté; peut-être parce que, en ayant souvent aperçu depuis, sans en manger, sur les tablettes des pâtissiers, leur image avait quitté ces jours de Combray pour se lier à d'autres plus récents; peut-être parce que, de ces souvenirs abandonnés depuis si longtemps hors de la mémoire, rien ne survivait, tout s'était désagrégé ; les formes - et celle aussi du petit coquillage de pâtisserie, si grassement sensuel sous son plissage sévère et dévot s'étaient abolies, ou, ensommeillées, avaient perdu la force d'expansion qui leur eût permis de rejoindre la conscience. Mais, quand d'un passé ancien rien ne subsiste, après la mort des autres, après la destruction des choses, seules, plus frêles mais plus vivaces, plus immatérielles, plus persistantes, plus fidèles, l'odeur et la saveur restent encore longtemps, comme des âmes, à se rappeler, à attendre, à espérer, sur la ruine de tout le reste, à porter sans fléchir, sur leur gouttelette presque impalpable, l'édifice immense du souvenir. »

Par ailleurs, il est difficile de considérer que les sens renvoient à la seule sensation. Sentir quelque chose via les sens s'accompagne automatiquement d'une interprétation ou, à défaut, d'une interrogation sur le quelque chose senti. La sensation qui serait uniquement le senti, est déjà idée de la sensation, donc traitement de celle-ci : quand je sens quelque chose, je sens immédiatement que je sens quelque chose et je le sais. On utilise ici je sens/je sais pour mettre en évidence l'interaction entre le physique et le mental : les terminaisons nerveuses (les papilles gustatives, les cellules du fond de l'œil) sont physiquement des terminaisons mais en tant que nerveuses elles ont leur fonction. Ces terminaisons ne donnent du sens que parce qu'elles sont en lien avec le système neuronal. Des cellules réceptrices non reliées à ce système ne donneraient pas du sens et n'auraient pas de sens.

Aussi, sentir, voir, entendre un objet urbain est-il déjà ressentir, immédiatement réinterprété par un système qui a sa dynamique propre et sa propre histoire. C'est la réception qui est faite de l'objet senti (en lien avec la nature de cet objet) qui fait impression. Robert Beck, parmi les auteurs des articles de ce dossier, va en ce sens dans la restitution qu'il fait de ce qui est senti/ressenti par F. C. Krieger ${ }^{1}$. Par exemple aussi, la sirène du premier mercredi midi de chaque mois que tous les habitants de France connaissent au point de ne plus vraiment l'entendre sera vécue émotionnellement de façon très forte, très stressante par un ressortissant étranger, nouvellement arrivé en provenance d'un pays vivant un conflit armé avec bombardements aériens prévenus par de tels signaux sonores. Cet exemple montre comment le ressenti dépend, individuellement, des aspects émotionnels, ici liés à la peur, elle-même inscrite dans le souvenir vivace d'un passé proche. Les articles qui suivent montrent comment se font ces réinterprétations, sur le mode émotionnel, mais aussi sur le mode hédoniste - entrée sur laquelle insiste Lucile Grésillon -, ou encore sur le mode cognitif, notamment quand l'enquêteur demande d'exprimer le ressenti, épreuve de parole qui mobilise aussi la connaissance et la rationalité renvoyant en même temps à la dimension passée de la trajectoire biographique individuelle, via ses souvenirs, et à la dimension future de cette trajectoire, via ses attentes et ses craintes qui, tous, au final, font que l'on voit, l'on sent, l'on entend... préférentiellement ce que l'on veut bien voir, sentir, entendre...

La ville, de son côté, au-delà des approches descriptives et analytiques, quantitatives comme qualitatives de sa réalité, fait l'objet d'un intérêt pour les représentations que les habitants, les usagers de cette ville se font d'elle. Le courant de la géographie des représentations associé au développement de la psychologie environnementale appliquée à l'espace urbain nécessite la compréhension, entre autres, du ressenti de la ville. La sensorialité intervient nécessairement, aussi, revisitée par la cognition et les jugements de valeur que cela implique : la ville, c'est beau; la ville pue; elle est bruyante... Le goût et le toucher sont moins sollicités par la ville et moins mobilisés par les chercheurs. Goûter la ville suppose l'action : contrairement aux sons, aux lumières et aux couleurs, le goût ne nous parvient pas sans un acte généralement volontaire puisque l'organe sensoriel est interne et la ville ne se pose pas sur la langue. De même, le sens du toucher est peu exploré avec la ville, plus que le goût qui

1. F. C. Krieger est un maître passementier bavarois qui tient un journal pendant un demi-siècle de 1821 à 1872 . 
est le grand absent de cet ensemble de textes, mais moins que l'odorat. Sans doute ce sens est-il quelque peu laissé de côté parce qu'il est généralement lié à l'action (mais ce n'est pas une action aussi spécifique que celle qui permet de goûter), au mouvement, plus qu'à la simple position ou au simple stationnement qui suffisent pour sentir, voir et entendre, même s'il est possible d'avoir ces sensations en mouvement et par le mouvement. En même temps, le toucher de la ville renvoie à peu d'interprétations sensibles au-delà du froid ou du rugueux, par exemple, qui qualifie plus l'objet que la sensation qui lui est liée : comme si le toucher était plus objectif que les autres sens, moins sujet à interprétation - ce qui ne signifie pas l'absence d'interprétation, l'absence de souvenirs mobilisés et caresser la pierre de Berchères, celle de la cathédrale de Chartres, mais aussi celle des bordures de trottoirs des rues voisines, n'est pas se frotter au granit armoricain. D'autre part, nous mettons en œuvre des systèmes de protection du corps, essentiellement les habits, alors qu'on ne peut s'astreindre durablement à fermer les yeux, se boucher les oreilles ou le nez, ainsi que des stratégies d'évitement. Le toucher, par l'action qu'il suppose, est, généralement, un acte volontaire, comme le goût mais à l'opposé des autres sens. Aussi ne touche-t-on de la ville que ce que l'on veut en toucher, il est donc précédé de la réflexion qui objective ce que l'on attend du toucher. Dans le cas contraire, c'est le choc, l'égratignure, l'écorchure, la piqure, la meurtrissure, la blessure. Le toucher, en lien avec la ville, lorsqu'il n'est pas volontairement recherché, est de l'ordre du « trop », et ce « trop » est plus facilement supportable lorsqu'il s'agit du bruit ou que cela sent vraiment trop mauvais, situations contre lesquelles on peut se défendre. Et il est rare que la ville soit trop difficile à regarder, à moins d'une luminosité intense. Pour le toucher, lorsqu'il y a choc, c'est déjà trop tard.

Au final, ce qui est questionné ici est, essentiellement, le quotidien, même si celui-ci renvoie nécessairement à l'extraordinaire, l'improbable, l'idéel, le fantasmatique : c'est par l'examen des pratiques spatiales, des pratiques de mobilité, dans une ville comprise à la fois comme un ensemble de possibles et une somme de contraintes avec lesquelles il faut faire, en lien avec leurs dimensions temporelles (par le souvenir et les attentes) qu'on atteint, par la mise en perspective du concret et de l'idéel, du réel et de l’idéal, le ressenti de la ville.
Les cinq textes qui sont présentés sont organisés ensemble de façon à montrer une progression dans la complexité, allant du plus sensoriel au plus réinterprété jusqu'aux sentiments et émotions, notamment. Cette progression révèle en fait des différences quant aux postures choisies relativement à l'intégration plus ou moins approfondie du sentir dans les différentes modalités du ressentir. Dans ces postures, on peut repérer deux types majeurs, qui marquent les bornes d'un gradient, dont l'un est la tentative visant à isoler le sens et, l'autre, à l'opposé, qui est la tentative d'intégrer les sens les uns aux autres et dans un système dynamique physiologique, neurologique, psychologique et social, pour aller du plus simple au plus complexe, et temporel, dont la temporalité est double : l'inscription du sentir dans les causes qui l'amènent et dans les conséquences en termes de ressentir et, d'autre part, l'inscription de ces conséquences dans la trajectoire biographique de l'individu, en tant qu'elles participent à la construction et à l'orientation, mêlant son sentir avec ses souvenirs et ses attentes. Ces deux tentatives sont vouées à l'échec, ces deux postures sont archétypales et, de ce fait, intenables : la première tant le sentir seul n'existe pas sans le ressentir, à moins de s'en tenir à une mesure purement physique (en décibels, en lux...) ou chimique (composition moléculaire...) qui, d'ailleurs, est plus de l'ordre de l'objet (sa nature, sa position relative, le contexte) que de l'ordre de l'individu sentant; la seconde tant l'ensemble est complexe et qu'aucune méthode ne parvient à saisir à la fois les phénomènes, processus et mécanismes en œuvre et leurs résultats, dans leur totalité, leur diversité et leur caractère mouvant. On se situe ainsi toujours entre ces deux pôles

L'entrée de Lucile Grésillon est, principalement, celle de l'olfaction, au départ de façon monosensorielle : comment la ville et plus précisément une station de RER sont-elles senties? Cependant, si le point de départ - prétexte et base d'une question de recherche qui envisage la complexité du couple formé du sentir et du ressentir - est clair, immanquablement l'information obtenue à partir de questionnaires rapides, in situ, renvoie à des objets, à des images dont la dimension visuelle efface le caractère olfactif, renvoie à des souvenirs mentalement représentés mais qui font plus image qu'odeurs ou parfums. L'auteure examine non seulement comment on sent (au sens le plus restrictif) les lieux urbains mais aussi comment 
l'olfaction mobilise nécessairement les autres sens, notamment la vue, ce qui ne devient évidence que lorsqu'il est demandé d'exprimer oralement ce qui est senti. Il faut y voir la pauvreté du vocabulaire relatif à l'odorat comparativement à celui lié à la vue mais aussi l'importance conférée au visuel, à la fois par la physiologie humaine et par la société. Plus encore, la dénomination des objets sentis se fait par le biais d'objets, utilisés apparemment de façon descriptive, mais qui réfèrent en fait, d'emblée, à des moments vécus ou imaginés, c'est-à-dire à ce qui relève de l'ambiance d'une cave par exemple où le caractère fermé du lieu renvoie à l'impression de renfermé de celui-ci, qui est bien de l'ordre de l'olfactif mais renvoie aussi au temps : le renfermé c'est, ainsi que l'indique le préfixe re-, ce qui est (en)fermé depuis longtemps. Un sens, y compris lorsqu'il est questionné de façon simple («qu'est-ce que, pour vous, cet endroit sent? ») renvoie nécessairement à ce qui est de l'ordre des souvenirs, des représentations, du vécu. En un mot à la personne. Sentir, c'est déjà ressentir.

Théa Manola examine l'espace par une approche multisensorielle, dont elle délivre les aspects méthodologiques fins et qui renvoient à la complexité des relations entre les sens, qui se renforcent, se complètent ou se nuancent sur le plan sensoriel comme sur le plan sensible. L'auteure renforce le texte précédent : la complexité du sentir n'est pas spécifique à l'olfaction, elle est consubstantielle à chaque sens, pris séparément et envisagé comme constitutif d'un système sensoriel. Dans ce texte aussi, on constate l'importance du visuel par rapport aux autres sens mais, en même temps, la relativité de cette importance tant la chercheuse est amenée, comme la précédente, à convoquer, sans en examiner les mécanismes mais parce qu'ils sont utilisés dans les discours qu'elle recueille, un vocabulaire sensible, sentimental, émotionnel et affectif qui dépasse la vue, l'ouïe, l'odorat...

Robert Beck envisage, lui, a contrario, le long terme textuel puisqu'il a étudié les 5500 pages du journal d'un maître passementier de Landshut (Bavière) écrit entre 1821 et 1872 . Cette analyse permet de mettre en relation la quotidienneté de ce qu'apportent les sens et le long terme d'un univers relativement stable, peu changeant qui est le cadre général des activités de ce F. C. Krieger, son travail, ses quelques loisirs et, plus encore, les relations sociales qu'il tisse dans sa ville, le sentiment d'appartenance à une communauté par la reconnais- sance d'une identité du lieu et du groupe desquels il est constitutif. Il est clair que la seule sensorialité ne permet pas tout cela. Mais les sens, notamment lorsqu'il s'agit de la vue de la ville dans sa globalité, c'est-à-dire la vue synthétique du paysage du quotidien, confortent ce sentiment d'appartenance, lui procurent une image qui vaut preuve, tout comme les changements liés à l'espace et donc les modifications de cette image sont la preuve d'une avancée de la société, de sa société. R. Beck, montre bien l'imbrication du rapport à l'environnement, du rapport à soi, à l'autre et à la communauté.

Benoît Feildel, l'auteur du cinquième texte, provoque une rupture : il ne part pas des sens pour en examiner les différentes dimensions, il part du rapport affectif à l'espace et montre comment celui-ci, par les différents mécanismes de son incessante construction, mobilise, entre autres, les sens, justement parce que sentir c'est déjà ressentir. Mais il inverse le propos : «Aussi le sentir n'est pas premier, c'est le ressentir - ce que produit l'affectivité - qui guide déjà notre manière d'éprouver sensoriellement notre environnement. » Envisageant la complexité du système formé des sens et de ce qu'ils apportent, préparé par le ressentir, lui-même relevant de la biographie personnelle, embrassant la sphère de l'affectivité, l'auteur montre l'intérêt qu'il y a de chercher à prendre en compte ce "sentir-ressentir ", à moins que ce ne soit un " ressentir-sentir » dans la construction volontaire des sociétés, à travers notamment son organisation spatiale.

Enfin, Denis Martouzet envisage, dans la continuité de ce qui précède, s'éloignant du sensoriel pour aborder de front l'affectif, de traiter le rapport affectif à l'espace, à la suite de plusieurs recherches menées précédemment sur cette question, par le biais d'une thématique transversale, celle de la liberté. Celle-ci ne fait pas l'objet d'une réflexion philosophique. Elle est mobilisée par l'auteur comme expression, recueillie lors d'enquêtes notamment, d'un ressenti, voire d'un sentiment : en quoi le sentiment de liberté, d'une part, mais aussi, d'autre part, la réalité d'une autonomisation de la personne par rapport à des figures d'autorité et par rapport à des contraintes matérielles renvoient-ils à la construction de l'affectivité de la personne? La complexité de cette question est illustrée par le cas, un peu énigmatique, un peu emblématique, d'un amoureux de la liberté : Jean-Jacques Rousseau. 